\title{
The Microbiota and Kidney Transplantation: Influence on the Graft
}

\author{
Authors: \\ *Maurizio Salvadori, ${ }^{1}$ Aris Tsalouchos ${ }^{2}$ \\ 1. Renal Unit, Department of Transplantation, Careggi University Hospital, \\ Florence, Italy \\ 2. Division of Nephrology, Santa Maria Annunziata Hospital, Florence, Italy \\ *Correspondence to maurizio.salvadori1@gmail.com \\ Disclosure: \\ The authors have declared no conflicts of interest. \\ Received: \\ 21.04 .21 \\ Accepted: \\ 16.07 .21 \\ Keywords: \\ Kidney transplant, kidney transplant outcomes, microbioma, microbiota, \\ pathobionts, pathogens. \\ Citation: \\ EMJ Urol. 2021;9[1]:95-106.
}

\section{Abstract}

The gut microbial community may be associated with complications after kidney transplantation. The indigenous microbiota has a significant and protective function that influences the transplant recipient response. Genetic or environmental factors may modify the indigenous microbiota and pathobionts appear.

In this condition, several disturbances of the kidney graft may be observed. These include acute rejection, infection, diarrhoea, disturbance in the induction of tolerance, and modification of immunosuppressive drug metabolism.

Recently, the use of prebiotics, probiotics, and synbiotics has been demonstrated to be effective in normalising these conditions and in restoring the generation of the normal indigenous microbiota.

An improved understanding of the function and composition of the indigenous microbiota may help in finding further solutions to stabilise the microbiota after kidney transplantation.

\section{INTRODUCTION}

The microbiota encompasses all of the microorganisms that live in specific niches of the body, including gut, skin, lungs, kidneys, bladder, and other organs. The term microbiota refers to bacteria, viruses, fungi, and archaea. This review principally discusses the bacteria living in the gastrointestinal tract, which have been more extensively studied and, in healthy conditions, live in the human body without damaging it. In particular, the authors examine how modifications to the microbiota that are known to be related

to several diseases, including renal diseases, may influence the outcomes of kidney transplantation.

\section{DEFINITIONS}

The terms microbiota and microbiome are often mutually used with the same meaning, but they have different significance.

Microbiota refers to all the microorganisms living in the human body. Recently, the total number of bacteria in the human body has been approximately evaluated to be $3.8 \times 10^{13} .{ }^{1}$ In healthy 
conditions, the resident microbiota is also called the indigenous microbiota. The composition and activity of the indigenous microbiota may be modified by genetic or environmental causes, leading to several diseases. In such conditions the indigenous microbiota are called pathobionts and the new condition is called dysbiosis.

Pathobionts are indigenous microbiota that are modified in their composition or activity and should be distinguished from pathogens, which are acquired infectious agents. ${ }^{2}$

The term microbiome refers to all the microbiota genes. $^{3}$ In normal conditions, the microbiome exerts important functions for the body, such as metabolic, structural, and protective functions.

\section{MICROBIOTA FUNCTIONS}

The principal functions exerted by the microbiome are metabolic, structural, and protective.

\section{Metabolic Function}

The metabolic function principally consists of vitamin and amino acid biosynthesis, bile acid biotransformation, and dietary fibre fermentation. ${ }^{4-6}$ The most important metabolic function is the production of short-chain fatty acids (SCFA) ${ }^{7}$

SCFA activate G protein-coupled receptors (GPR) including GPR41, GPR43, and GPR109A. The binding of SCFA to their receptors, which are broadly expressed in a variety of human cells and tissues such as colonic epithelial cells, immune cells, adipocytes, hepatocytes, cardiomyocytes, and renal epithelial cells, regulates metabolic syndromes such as obesity and Type 2 diabetes $^{8}$ and regulates energy homeostasis, stimulating glucagon-like peptide 1 secretion and inhibiting atherosclerosis progression. ${ }^{9}$ In addition, the binding to olfactory receptor 78 (Olfr78) has beneficial effects on arteriole blood pressure (Figure 1). 10,11 Moreover, SCFA through regulation of the immune system and cytokine production ${ }^{12}$ regulate the balance between $T$ regulatory cells (Treg) and Th17, inducing an anti-inflammatory effect. In the case of pathobiont prevalence, dysbiosis is produced and such beneficial effects on the immune system are interrupted, resulting in conditions that favour inflammatory disease.

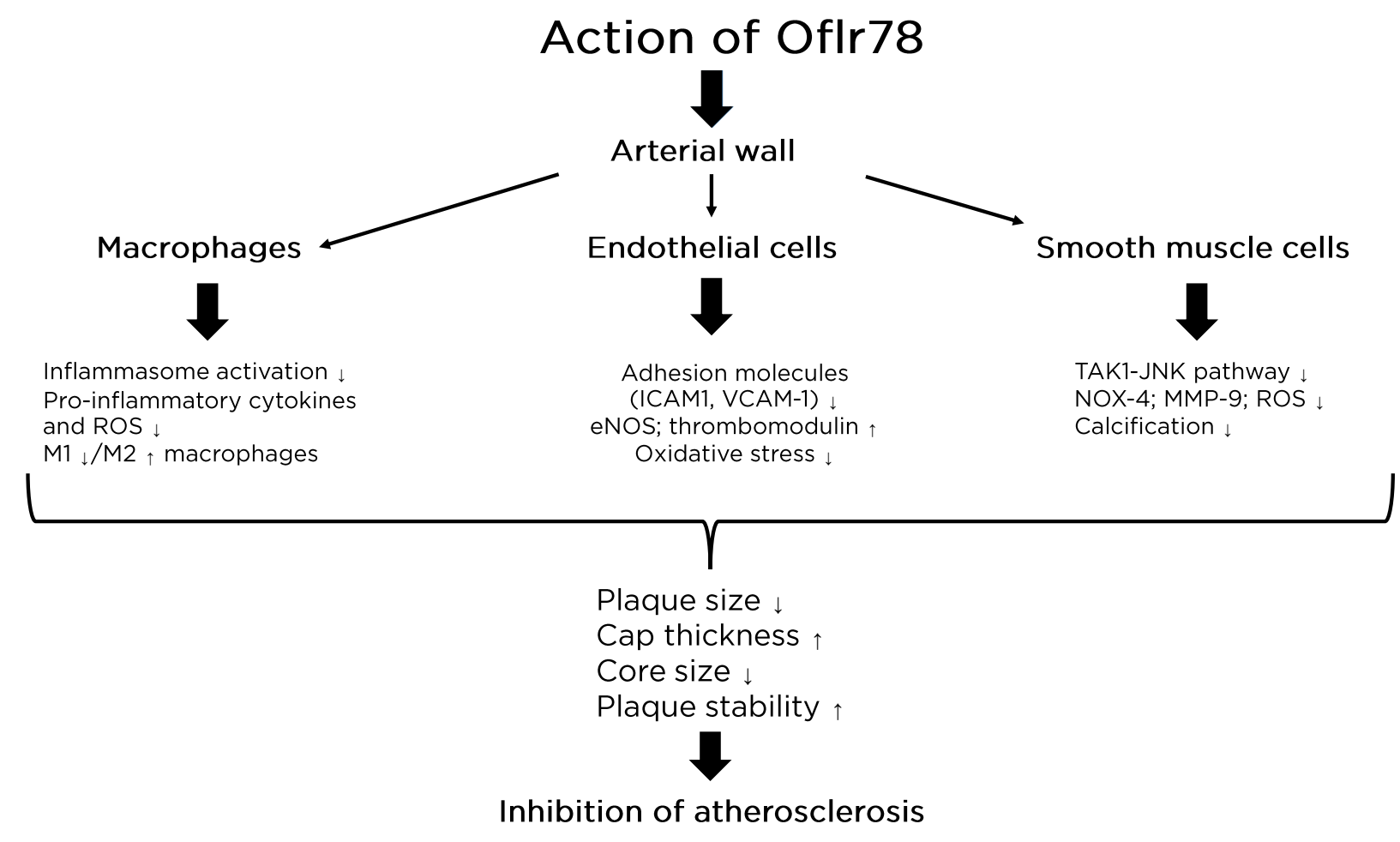

Figure 1: Action of OfIr78 on the vascular system.

eNOS: endothelial nitric oxide synthase; ICAM1: intercellular adhesion molecule-1; NOX 4: NADPH oxidase; Olfr78: olfactory receptor 78; ROS: reactive oxygen substances; VCAM-1: vascular cell adhesion molecule-1. 


\section{Structural Function}

The indigenous microbiota regulates the mucus layer property of the gut, normal crypt and villi development, villi vascularisation, and tight junction regulation.

This is essential to down-regulate the back diffusion of cytokines produced in the gut. When pathobionts or pathogens such as Escherichia coli, Clostridium difficile, and C. perfrigens prevail, a condition of dysbiosis occurs and the back diffusion of cytokines such as IL-4, IL-1B, TNF-a, and IFN-y is increased. ${ }^{13}$

\section{Protective Function}

The protective function is composed of a three-layered system: a barrier fortification exerted by mucin glycoproteins that form a layer of the gut epithelia that prevents bacterial adhesion; antimicrobial peptides (AMP) secreted in normal conditions by the epithelial cells, as $\alpha$ and $\beta$ defensins and other substances; ${ }^{14,15}$ and the possibility of activating innate and adaptive immunity. This fact is principally due to dendritic cells that take up bacteria and induce B cells to differentiate into IgA plasma cells that secrete IgA. ${ }^{16}$

In addition, the indigenous microbiota induces CD4+ cells to differentiate into four main subtypes: Th1, Th2, Th17, and Treg, and contributes to normalising the ratio of these subtypes. An important role is exerted by segmental filamentous bacteria, which induce the growth and differentiation of Th17 and Th1 cells. ${ }^{17}$ Clostridia also promote the accumulation of Tregs and the production of IL-10, which exerts anti-inflammatory effects. II-10 and Tregs are also induced by Bacteriodes fragilis. ${ }^{18}$

When the indigenous microbiota is altered by any condition, pathobionts appear and dysbiosis occurs, altering the relationship between the microbiota and the immune system, leading to several diseases.

\section{MICROBIOTA AND BIOLOGICAL SYSTEMS}

In healthy subjects, the indigenous microbiota provides several benefits to different biological systems. Indeed, the indigenous microbiota affects the host by the production of metabolites and gut neuropeptides. As a consequence, it exerts control over many important functions such as mood, immune response, digestion, and heart rate. A bidimensional communication between the gut, its microbioma, and the nervous and neuroendocrine systems is established. ${ }^{19}$ Changes in the composition of the intestinal bacterial community may result in dysbiosis, which contributes to triggering various diseases in almost all the biological systems. This imbalance of intestinal microbiota homeostasis may alter commensal bacteria and host metabolism, as well as immune function. Dysbiosis also causes an increase in intestinal permeability due to exposure to molecular patterns, leading to a chronic inflammatory process that can result in diseases in all biological systems. ${ }^{20}$

Of particular interest is the relationship between indigenous microbiota, dysbiosis, and the kidney.

\section{INTESTINAL MICROBIOTA AND THE KIDNEY}

An inter-relationship between the gut and the kidney occurs either by the activation of the immune system or by the microbiotaderived metabolites. While the indigenous resident microbiota induces a normal balance between Treg and Th17 cells, pathobionts may activate Th17 cells and favour renal inflammation and injury. ${ }^{21}$

Similarly, microbiota-derived metabolites may affect kidney function. The protective role of SCFA has been already highlighted. In addition, SCFA have beneficial effects by reducing the production of cytokines and chemokines such as IL- $\beta$, IL-6, TNFa, and monocyte chemoattractant protein. ${ }^{22}$

On the contrary, pathobionts such as E. coli have deleterious effects. The phenomenon is bilateral. Indeed, dysbiosis may facilitate acute kidney injury (AKI) by modifying SCFA composition and generating high quantities of toxic indoxylsulfate and trimethylamine $\mathrm{N}$ oxide (TMAO). This fact may favour the transition from $A K I$ to chronic kidney disease (CKD). On the other hand, AKI may modify the gut bacterial composition (Figure 2). ${ }^{23}$ 


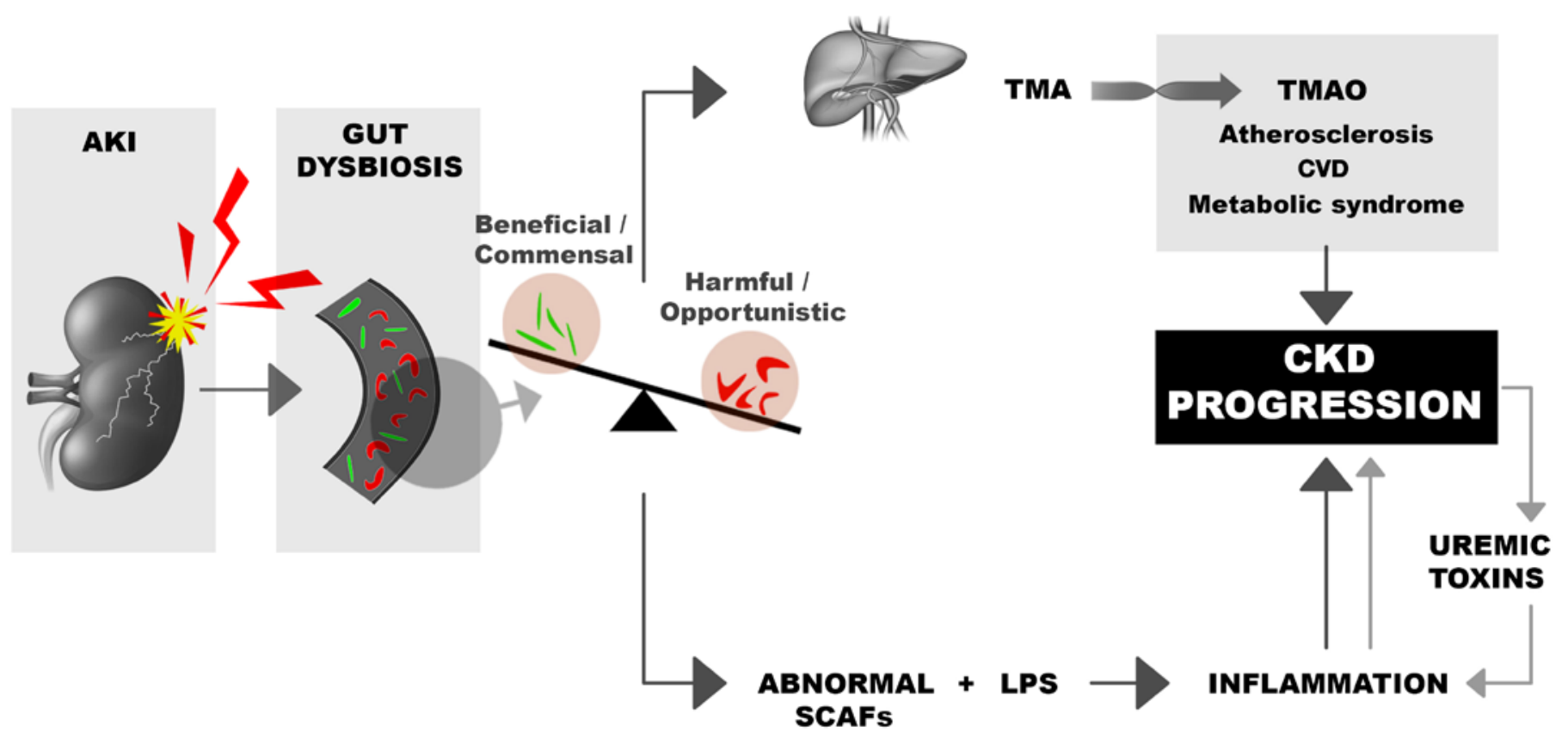

Figure 2: Relationship between acute kidney injury, chronic kidney disease, and toxic substances.

Dysbiosis may facilitate AKI by modifying the SCFA composition and generating high quantities of toxic indoxysulfate and TMAO.

AKI: acute kidney injury; CKD: chronic kidney disease; CVD: cardiovascular disease; LPS: lipopolysaccharides; SCFA: short-chain fatty acids; TMA: trimethylamine; TMAO: trimethylamine $\mathrm{N}$ oxide.

Overall, the association of $\mathrm{AKI}$ and pathobionts may favour atherosclerosis, cardiovascular diseases, inflammation, and CKD progression.

Dysbiosis in patients with end-stage kidney disease (ESKD) has been observed in several studies $^{24}$ that found the presence of pathobionts and a huge difference between the gut microbiota composition in healthy subjects and that of patients with ESKD.

\section{MICROBIOTA AND RENAL TRANSPLANTATION}

\section{Microbiota Composition,}

\section{Pre-/Post-transplant Dysbiosis, and} Effect on the Immune System

After kidney transplantation, gut microbiota composition further modifies, principally in the first period after transplantation. Indeed, in this period, kidney transplant patients are receiving high doses of immunosuppressants to avoid rejection and chemoprophylactic therapy to avoid infection. Both immunosuppressants and antibiotics modify indigenous microbiota and favour the appearance of pathobionts. Other factors involved in the modification of gut microbiota after kidney transplantation are the use of proton pump inhibitors, the use of mycophenolate mofetil (MMF), and a low estimated glomerular filtration rate (eGFR). In a recent study by Swarte et al., ${ }^{25}$ faecal samples of 139 kidney transplant recipients were compared with 105 healthy controls. The aforementioned factors (immunosuppressant assumption and antibiotics) were significant determinants in the modification of gut microbiota. In a study from Lee et al. $^{26}$ using polymerase chain reaction in 26 kidney transplant recipients, an important change in microbiota composition was documented in samples obtained in the pre- and post-transplant period. There was a decrease in Firmicutes and an increase of Actinobacteria and Proteobacteria (Table 1). In a different study of 142 kidney transplant recipients, ${ }^{27}$ pathogens such as $C$. difficile and $E$. coli were found in $30 \%$ of patients.

Major changes in the microbiota composition occur in the first month post-transplant. ${ }^{28,29}$ 
Table 1: Alterations in the gut microbiota following kidney transplantation, according to phylum and order.

\begin{tabular}{|l|l|l|}
\hline Phylum & Pre-transplantation cohort (\%) & Post-transplantation cohort (\%) \\
\hline Firmicutes & 91.8 & 87.7 \\
\hline Actinobacteria & 2.0 & 7.6 \\
\hline Proteobacteria & 0.9 & 4.1 \\
\hline Bacteroidetes & 2.8 & 0.6 \\
\hline Order & & \\
\hline Clostridiales & 64.8 & 64.3 \\
\hline Lactobacillales & 19.1 & 12.0 \\
\hline Erysipelotrihales & 5.6 & 10.2 \\
\hline Bifidobacteriales & 1.6 & 6.6 \\
\hline Enterobacteriales & 0.4 & 3.9 \\
\hline Bacteroidales & 2.8 & 0.6 \\
\hline
\end{tabular}

Table 2: Studies of post-transplant interactions between microbiota modifications and different outcomes.

\begin{tabular}{|c|c|c|c|c|}
\hline Author & Year & Patients (n) & Aim of the study & Main findings \\
\hline Swarte et al. ${ }^{25}$ & 2020 & 139 KTR; 105 controls & $\begin{array}{l}\text { Characterisation } \\
\text { of pre- and post- } \\
\text { transplant microbiota }\end{array}$ & $\begin{array}{l}\text { Reduction of } \\
\text { Firmicutes }\end{array}$ \\
\hline Fricke et al. ${ }^{29}$ & 2014 & $60 \mathrm{KTR}$ & $\begin{array}{l}\text { Characterisation } \\
\text { of pre- and post- } \\
\text { transplant microbiota }\end{array}$ & $\begin{array}{l}\text { Dysbiosis occurs in } \\
\text { the first month after } \\
\text { transplant }\end{array}$ \\
\hline Lee et al. ${ }^{26}$ & 2014 & 26 KTR & $\begin{array}{l}\text { Characterisation } \\
\text { of pre- and post- } \\
\text { transplant microbiota }\end{array}$ & $\begin{array}{l}\text { Decrease of } \\
\text { Firmicutes; } \\
\text { Increase in } \\
\text { Actinobacteria and } \\
\text { Proteobacteria }\end{array}$ \\
\hline Westblade et al. ${ }^{27}$ & 2019 & 142 KTR & $\begin{array}{l}\text { Characterisation } \\
\text { of pre- and post- } \\
\text { transplant microbiota }\end{array}$ & $\begin{array}{l}\text { Presence of } \\
\text { Clostridium difficile } \\
\text { and Escherichia coli }\end{array}$ \\
\hline Wang et al. ${ }^{28}$ & 2015 & NA & $\begin{array}{l}\text { Characterisation } \\
\text { of pre- and post- } \\
\text { transplant microbiota }\end{array}$ & $\begin{array}{l}\text { Dysbiosis occurs in } \\
\text { the first month after } \\
\text { transplant }\end{array}$ \\
\hline \multicolumn{5}{|c|}{ Dysbiosis and acute rejection incidence } \\
\hline Lee et al. ${ }^{26}$ & 2014 & $26 \mathrm{KTR}$ & Incidence of AR & $\begin{array}{l}3 \text { patients with } \\
\text { AR had different } \\
\text { microbiota }\end{array}$ \\
\hline Fricke et al. ${ }^{29}$ & 2014 & $60 \mathrm{KTR}$ & Incidence of AR & $\begin{array}{l}\text { Reduction in } \\
\text { Firmicutes in patients } \\
\text { with late AR }\end{array}$ \\
\hline Carron et al. ${ }^{35}$ & 2019 & 146 KTR & Incidence of AR & $\begin{array}{l}39 \% \text { of } A R \text { associated } \\
\text { with increase } \\
\text { of inflammation } \\
\text { biomarkers }\end{array}$ \\
\hline Wang et al. ${ }^{36}$ & 2021 & $\begin{array}{l}24 \text { KTR with AMR; } \\
29 \text { KTR without AMR }\end{array}$ & Incidence of AMR & $\begin{array}{l}\text { AMR associated with } \\
\text { Clostridiales }\end{array}$ \\
\hline
\end{tabular}


Table 2 continued.

\begin{tabular}{|c|c|c|c|c|}
\hline \multicolumn{5}{|c|}{ Dysbiosis and infection incidence } \\
\hline Fricke et al. ${ }^{29}$ & 2014 & $60 \mathrm{KTR}$ & $\begin{array}{l}\text { Incidence of urinary } \\
\text { infections }\end{array}$ & $\begin{array}{l}\text { Higher incidence of } \\
\text { UTI when Firmicutes } \\
\text { reducted }\end{array}$ \\
\hline Lee et al. ${ }^{26}$ & 2014 & $26 \mathrm{KTR}$ & Incidence of UTI & $\begin{array}{l}\text { Faecal abundance } \\
\text { of Enterococcus } \\
\text { associated with UTI }\end{array}$ \\
\hline Lee et al. ${ }^{40}$ & 2019 & $168 \mathrm{KTR}$ & $\begin{array}{l}\text { Incidence of viral } \\
\text { infection }\end{array}$ & $\begin{array}{l}\text { High butyrate- } \\
\text { producing bacteria } \\
\text { associated with less } \\
\text { viral infection }\end{array}$ \\
\hline \multicolumn{5}{|c|}{ Dysbiosis and interstitial fibrosis } \\
\hline Modena et al. ${ }^{42}$ & 2017 & $\begin{array}{l}25 \text { KTR with fibrosis; } \\
25 \text { KTR without } \\
\text { fibrosis }\end{array}$ & $\begin{array}{l}\text { Dysbiosis of UTI and } \\
\text { fibrosis }\end{array}$ & $\begin{array}{l}\text { Presence of } \\
\text { pathobionts } \\
\text { associated with } \\
\text { fibrosis }\end{array}$ \\
\hline \multicolumn{5}{|c|}{ Dysbiosis and diarrhoea } \\
\hline Lee et al. ${ }^{26}$ & 2014 & $26 \mathrm{KTR}$ & Incidence of diarrhoea & $\begin{array}{l}\text { Diarrhoea associated } \\
\text { with reduction of } \\
\text { Ruminococcus, Dorea, } \\
\text { and Coprococcus }\end{array}$ \\
\hline Lee et al. ${ }^{44}$ & 2019 & $71 \mathrm{KTR}$ & Incidence of diarrhoea & $\begin{array}{l}\text { Diarrhoea associated } \\
\text { with increase of } \\
\text { Lachnoclostridium, E. } \\
\text { coli, and Enterococcus }\end{array}$ \\
\hline Zhang et al. ${ }^{46}$ & 2021 & $97 \mathrm{KTR}$ & Incidence of diarrhoea & $\begin{array}{l}\text { Diarrhoea associated } \\
\text { with higher faecal } \\
\text { B-glucoronidase } \\
\text { activity }\end{array}$ \\
\hline \multicolumn{5}{|c|}{ SCFA after kidney transplant and tolerance } \\
\hline Lee et al. ${ }^{40}$ & 2019 & $168 \mathrm{KTR}$ & $\begin{array}{l}\text { Butyrate-producing } \\
\text { bacteria and infection }\end{array}$ & $\begin{array}{l}\text { Higher infection } \\
\text { incidence associated } \\
\text { with low butyrate } \\
\text { levels }\end{array}$ \\
\hline Poesen et al. ${ }^{51}$ & 2016 & 51 KTR & $\begin{array}{l}\text { Uraemic toxins after } \\
\text { transplant }\end{array}$ & $\begin{array}{l}\text { Uraemic toxin levels } \\
\text { lower after transplant }\end{array}$ \\
\hline Colas et al..$^{50}$ & 2019 & $113 \mathrm{KTR}$ & Induction of tolerance & $\begin{array}{l}\text { Presence of } \\
\text { Proteobacteria } \\
\text { favours tolerance }\end{array}$ \\
\hline \multicolumn{5}{|c|}{ Dysbiosis and immunosuppressants } \\
\hline Lee et al. ${ }^{53}$ & 2015 & $19 \mathrm{KTR}$ receiving TAC & $\begin{array}{l}\text { Need to increase TAC } \\
\text { dosing }\end{array}$ & $\begin{array}{l}\text { Patients with } \\
\text { high levels of } \\
\text { Faecalibacterium } \\
\text { prausnizii needed } \\
\text { higher doses of TAC }\end{array}$ \\
\hline Zheng et al. ${ }^{52}$ & 2019 & $\begin{array}{l}260 \text { KTR receiving } \\
\text { TAC, treated or not } \\
\text { with antibiotics }\end{array}$ & $\begin{array}{l}\text { Need to increase TAC } \\
\text { dosing }\end{array}$ & $\begin{array}{l}\text { Patients receiving } \\
\text { antibiotics had } \\
\text { dysbiosis and needed } \\
\text { higher TAC doses }\end{array}$ \\
\hline
\end{tabular}




\begin{tabular}{|l|l|l|l|l|}
\hline Gibson et al. ${ }^{55}$ & 2021 & Review of 75 articles & $\begin{array}{l}\text { Change in } \\
\text { microbiome due to } \\
\text { immunosuppressants }\end{array}$ & $\begin{array}{l}70 \% \text { of the articles } \\
\text { indicated changes } \\
\text { in quantities } \\
\text { of anaerobic } \\
\text { bacteria including } \\
\text { Ruminococcaceae, } \\
\text { Lachnospiraceae, } \\
\text { Firmicutes, } \\
\text { Bacteroides, and } \\
\text { Clostridiales }\end{array}$ \\
\hline
\end{tabular}

AR: Acute rejection; AMR: antibody-mediated rejection; KTR: kidney transplant recipient; NA: not available; SCFA: short-chain fatty acids; TAC: tacrolimus; UTI: urinary tract infection.

The dysbiosis related to the imbalance between indigenous microbiota and pathobionts has relevant metabolic and clinical consequences on different post-transplant outcomes such as acute rejection, acute infection, interstitial fibrosis, post-transplant diarrhoea, reduced production of SCFA, and abnormalities in immunosuppressant levels. Table 2 shows all the principal studies that have been conducted on post-transplant dysbiosis and its consequences.

The table clarifies the more important studies in these fields, according to the different variables taken into consideration. All the cited studies have been conducted in humans, and it is not surprising that sometimes the same study was conducted to explore different outcomes simultaneously.

\section{Post-transplant Dysbiosis and the Immune System}

Post-transplantation complications are closely associated with the host immune system. There is also an interaction between a person's gut microbiota and immune system. Animal and human studies have shown that gut microbial population and diversity are altered after allogeneic transplantation. Moreover, when complications such as infection or rejection occur, gut microbial populations and diversity present a significant dysbiosis. ${ }^{28}$ Different factors, including immunosuppression and antibiotic therapy, lifestyle, and diet may alter the microbiota and lead to dysbiosis in kidney transplant patients.
Dysbiosis disrupts gut epithelial barrier, loses barrier integrity, and leads to overgrowth of pathogens. Leaky gut and increased permeability allow translocation of bacteria and their components into the inner environment. ${ }^{30}$ In this dysbiosis situation, the pro-inflammatory response triggers to eliminate pathogens by intestinal epithelial cells and IL- $1, \mathrm{IL}-6$, and IL-18 secretion by dendritic cells (DC) and by macrophages that induce development of the effector CD4+ T cells Th1 and Th17. The innate immune response leads to a state of systemic and allograft inflammation. ${ }^{31}$ Moreover, dysbiosis decreases the regulatory $T$ cells and increases the effector $T$ cells that beside activated innate immunity boost adaptive immunity. ${ }^{32}$ These immune responses can preserve the activation of alloreactive $T$ cells by cross-reacting with commensal organisms and molecular mimicry, leading to graft rejection. On the other hand, in the colon and liver dysbiotic gut-derived uraemic toxins (e.g., phenols and indoles) are further metabolised to TMAO, p-cresyl sulfate (PCS), and indoxyl sulfate (IS) and leaked into the systemic circulation. Accumulation of PCS in kidney tubular cells generates reactive oxygen species (ROS) that lead to the production of inflammatory cytokines and profibrotic factors, resulting in cell injury. Moreover, through special receptors localised on the basolateral membrane of renal proximal tubular cells, IS induces inflammation and nephrotoxicity. ${ }^{33}$

The main consequences of dysbiosis in kidney transplant patients are a higher 
incidence of acute rejections, acute infections, interstitial fibrosis, post-transplant diarrhoea, reduced production of protective agents such as SCFA by the gut microbiota and reduced tolerance, and modification of immunosuppressant levels in the blood.

\section{Dysbiosis and Acute Rejection}

In vivo studies and research in humans have documented that post-transplant dysbiosis is associated with clinically significant complications such as graft rejection. ${ }^{26,34}$ Lee et al. $^{26}$ performed an excellent study to clarify the alterations of gut microbiota in patients pre- and post-kidney transplant. They used the polymerase chain reaction amplification of the 16S, RNA V4-V5 variable region to analyse the bacterial composition of faecal specimens from 26 kidney transplant recipients during the first 3 months of transplantation. The study documented significant differences between the groups with and without acute rejection (AR), with Bacteroidetes being lower at the phylum level in the AR group compared to the no-AR group, while Lactobacillales, Enterococcus, Anaerofilum, and C. tertium were higher in the AR group at the order level.

In a recent study ${ }^{29}$ the microbiota was evaluated pre- and post-transplant in 60 renal transplant recipients. Samples were obtained from urine, oral swabs, rectal swabs, and blood for up to 6 months after transplantation.

Carron et al. ${ }^{35}$ found in 146 kidney transplant recipients that $39 \%$ of those experiencing AR had increased inflammation biomarkers in the blood.

The most relevant changes in the microbiota were principally observed in the first month after transplantation. Rejection episodes were correlated with a significant decrease in Anaerotruncus, Coprobacillus, and Coprococcus (all from phylum Firmicutes) in patients with late acute rejection, while significant changes in Leptotrichia, Neisseria, and Actinobacteria were observed in patients with early rejection. The study also documented that pre-transplant microbiota differences can be correlated with post-transplant events, suggesting that specific pre-transplant features of microbiota can act as diagnostic biomarkers in predicting graft outcomes.
In a very recent study, Wang et al. ${ }^{36}$ characterised the gut microbiota possibly associated with antibody-mediated rejection (AMR) in 24 kidney transplant recipients with AMR. The study showed the gut microbial community of kidney transplant recipients with AMR was different from controls and that Clostridiales is a potential marker to distinguish recipients with AMR.

\section{Dysbiosis and Infection}

Recent studies suggested that the urinary tract is characterised by a unique specific urinary microbiota, different from that of the gut. In addition to change ${ }^{37,38}$ the microbiota composition may be critical for the development of urinary tract infection and differences have been observed between patients and healthy subjects. ${ }^{39}$

Change in the urinary microbiota may cause urinary infections also in transplant patients. In kidney transplant patients, in addition to change in the urinary microbiota, modifications in the gut microbiota may generate infections. Risk factors to generate dysbiosis in kidney transplant recipients are dietary patterns, changes to colonic and bowel transit time, immunosuppression, antibiotics, and lifestyle. All these factors may induce an increased bacterial translocation, an increased metabolic endotoxinaemia, and an increased formation of microbial toxins. ${ }^{34}$

The aforementioned study on acute rejection ${ }^{29}$ documented that similar changes in the microbiota were also associated with a higher incidence of urinary tract infections. In particular, the abundance of the genus Anaerotruncus (phylum Firmicutes) was markedly decreased in respect to other patients.

A high incidence of urinary and gastrointestinal infections was also reported in the studies by Lee et al $^{26}$ and Chan et al. ${ }^{34}$ In a different study, Lee et al. ${ }^{40}$ found in 168 kidney transplant recipients that the presence of high butyrate-producing bacteria was associated with a reduced number of viral infections. In a recent study, ${ }^{41}$ a transplant patient with recurrent urinary infections recovered after faecal microbiota transplantation (FMT), which induced a marked decrease in the abundance of E. coli in the urinary microbiota. 


\section{Dysbiosis and Renal Fibrosis}

In a recent study on transplant patients, Modena et al. ${ }^{42}$ collected urinary samples from 25 patients after kidney transplantation. All of these patients developed interstitial fibrosis/tubular atrophy (IF/TA) at 6 months after transplantation at kidney biopsy. Patients were compared with 23 kidney transplant recipients who did not develop IF/TA. Patients with IF/TA had a decreased number of Lactobacillus and Streptococcus genera. The authors concluded that modification of the urinary microbiota could develop IF/TA by altering the host immune response.

\section{Dysbiosis and Diarrhoea}

Diarrhoea frequently occurs in kidney transplant patients and its aetiology is often not recognised. With the exception of patients whose diarrhoea may be ascribed to a specific infection and the presence of pathogens, in approximately 85\% of transplanted patients with diarrhoea the diagnosis is missing. Diarrhoea has been ascribed to the use of MMF, but the discontinuation of MMF is dangerous for the risk of rejection.

In the already-mentioned study by Lee et al. ${ }^{26}$ of 26 kidney transplant patients affected by diarrhoea, a reduction of commensal indigenous microbiota, such as Ruminococcus, Dorea, and Coprococcus, was observed without detecting pathogens such as $C$. difficile. Similar data were found by Xiao et al. ${ }^{43}$ In a more recent study in faecal specimens, Lee et al. ${ }^{44}$ found a reduction in genera similar to the previous study. In addition, the authors found a significant increase in genera Lachnoclostridium, E. coli, and Enterococcus. The genera that are reduced in the patients with diarrhoea develop in normal conditions, metabolic functions essential for the healthy condition. As a consequence, these functions are lacking during diarrhoea. ${ }^{45} \mathrm{~A}$ very recent study by Zhang et al. ${ }^{46}$ confirmed these data in 97 kidney transplant patients with diarrhoea. In this study, diarrhoea was associated with higher faecal $\beta$-glucuronidase.

The conclusion of all these studies is that gut dysbiosis, rather than the presence of pathogens or the use of MMF, represents the principal cause of post-transplant diarrhoea in the majority of patients. In addition, in several studies ${ }^{47}$ FMT was effective in controlling post-transplant diarrhoea.

\section{Short-Chain Fatty Acids in Kidney Transplantation and Tolerance}

Several studies in animals have documented the beneficial effects of SCFA produced by gut indigenous microbiota and the damage that occurs in the case of reduction of SCFA, as happens post-transplant. ${ }^{48,49}$

Lee et al., ${ }^{40}$ in a study of 168 kidney transplant recipients, documented the beneficial effects of butyrate-producing bacteria (BPG) and a higher incidence of infection in transplanted patients with low levels of butyrate. In an interesting study conducted in mice after transplantation, $\mathrm{Wu}$ et al. ${ }^{48}$ documented a donor-specific tolerance related to high levels of Tregs induced by SCFA. In a different study, ${ }^{50}$ tolerance was related to a Proteobacteria profile that included Janthinobacterium, Clostridia, and Firmicutes. The authors concluded that microbiota may favour the tolerance state that may be inhibited by the use of immunosuppressants. In another study, Poesen ${ }^{51}$ documented that uraemic toxins are lower post-transplant and this could have a favourable effect on tolerance.

\section{Interactions Between the Microbiota and Immunosuppressive Drugs}

Factors such as age, sex, race, and CYP3A5 polymorphisms influence the absorption and metabolism of calcineurin inhibitors (CNI). Recently, the indigenous microbiota or the presence of pathobionts have been documented to exert an important role in $\mathrm{CNI}$ metabolism. ${ }^{52}$

Lee et al. ${ }^{53}$ examined the role of microbiota on CNI metabolism. In their study, the authors observed 19 patients receiving tacrolimus (TAC) as immunosuppressive treatment. There were two groups of patients: patients needing to receive increasing TAC doses to achieve the optimal blood level, and patients whose TAC levels were stable over time, with no need to adjust the dosage. By examining the microbiota, patients needing higher doses had a high level of Faecalibacterium prausnizii in the gut that was the most significant factor among those influencing TAC metabolism.

In a different study, Guo et al. ${ }^{54}$ found in vitro that $F$. prausnizii was able to produce a TAC metabolite with less immunosuppressive activity. In the same study, the same metabolite was 
found in the stool of patients treated with TAC and with high gut levels of F. prausnizii. Similarly, Clostridia and Bacteroidales were also found to be able to produce inactive metabolites.

The conclusions of these studies were that different microbiota or pathobionts may influence the TAC exposure in kidney transplant patients. On one hand, the microbiota may alter the metabolism of immunosuppressants; on the other hand, immunosuppressants may alter the gut indigenous microbiota, as documented in a review by Gibson et al. ${ }^{55}$ The authors reviewed 75 articles and observed that the major part of the studies indicated that immunosuppressants induce modifications in pathobionts mostly modifying anaerobic bacteria, including Firmicutes and Bacteroides.

\section{Microbial Therapies in Kidney Transplantation}

The treatment of gut dysbiosis can be divided into probiotics, prebiotics, symbiotics, and FMT. Probiotics are defined by the World Health Organization (WHO) as live organisms that, when administered in adequate amounts, confer a health benefit to the host. ${ }^{56}$ They have the characteristics to be able to survive the harsh conditions of the digestive tract, to proliferate in the lower gastrointestinal tract, to be stable, and to have a positive healthy effect in human studies. ${ }^{57}$ Probiotics compete with pathogens for adhesion to the gastrointestinal epithelium, inhibit the production of bacterial toxins, and produce their own antimicrobial substances. ${ }^{58}$ Plain yoghurt, cottage cheese, and vinegar are among the substances containing probiotics. Probiotics such as Lactobacilli and Streptococci have been used principally in liver transplantation, ${ }^{59-61}$ documenting significant activity in reducing infection rates.

Prebiotics are defined as a non-viable food component that confers health benefits on the host associated with modulation of the microbiota. Prebiotics must be resistant to the actions of acid in the stomach, bile salts, and other enzymes in the intestines and should not be absorbed by the upper gastrointestinal tract. They act by producing SCFA, mucin, and increasing IgA production. To date, only insulin and trans-galacto-olisaccharides may be considered probiotics. ${ }^{62}$
Symbiotics are a combination of prebiotics and probiotics. An example is the combination of Lactobacillus rhamnosus and insulin. ${ }^{63}$

The principal limitation of the use of these compounds is that many have been studied in animals or in human liver transplantation or in diseases different from transplantation. In addition, the tolerability of prebiotics and probiotics in the transplant population has not been adequately assessed.

FMT is a promising option for a range of disorders including transplant disorders sustained by $C$. difficile. ${ }^{64}$ The efficacy of FMT in kidney transplantation is, to date, documented by case reports. Henig et al. ${ }^{65}$ recently reported the efficacy of FMT in stem cell transplantation.

\section{FUTURE PERSPECTIVES}

The two main new perspectives are the search for new therapies and an improved knowledge of gut microbiota and pathobionts. Lubiprostone, a synthetic derivative of prostaglandins, produced an improvement in the microbiota profile of a rat model.

Similarly, the trimethylamine inhibitor 3,3-dimethyl-1-1 butanol was shown to inhibit atherosclerotic lesions in mice. ${ }^{66}$ An improved understanding of microbiota could be possible by the use of sequencing techniques and the application of metabolomics.

\section{CONCLUSIONS}

In the case of kidney transplantation, microbiota and kidney transplant have a reciprocal 'doubleedged sword' action. After transplantation, because of the immunosuppressive drugs and of prophylactic antibiosis, the gut indigenous profile modifies, particularly in the first month after transplantation. This modification may influence the graft outcomes, causing acute rejection, infection, renal fibrosis, and modification of the drug metabolism, immunosuppressants included. It is possible to modify an abnormal microbiota with the use of prebiotics, probiotics, and diet modification. It should be highlighted that there are few studies referring to the microbiota in renal transplantation and they refer to a small number 


\section{References}

1. Sender $L$ et al. Revised estimates for the number of human and bacteria cells in the body. PLoS Biol. 2016;14(8):e1002533.

2. Chow $\mathrm{J}$ et al. Pathobionts of the gastrointestinal microbiota and inflammatory disease. Curr Opin Immunol. 2011;23(4):473-80.

3. Bäckhed F et al. Dynamics and stabilization of the human gut microbiome during the first year of life. Cell Host Microbe. 2015;17(5):690703.

4. Anderson JW et al. Health benefits of dietary fiber. Nutr Rev. 2009;67(4):188-205.

5. Lombard $\mathrm{V}$ et al. The carbohydrateactive enzymes database (CAZy) in 2013. Nucleic Acids Res. 2014;42(Database issue):D490-5.

6. Louis P, Flint HJ. Diversity, metabolism and microbial ecology of butyrate-producing bacteria from the human large intestine. FEMS Microbiol Lett. 2009;294(1):1-8.

7. Rowland I et al. Gut microbiota functions: metabolism of nutrients and other food components. Eur J Nutr. 2018;57(1):1-24.

8. Ichimura A et al. Free fatty acid receptors as therapeutic targets for the treatment of diabetes. Front Pharmacol. 2014:5:236.

9. Lukasova M et al. Nicotinic acid (niacin): new lipid-independent mechanisms of action and therapeutic potentials. Trends Pharmacol Sci. 2011;32(12):700-7.

10. Pluznick J. A novel SCFA receptor, the microbiota, and blood pressure regulation. Gut Microbes. 2014:5(2):202-7.

11. Kiepura A et al. Anti-atherosclerotic potential of free fatty acid receptor 4 (FFAR4). Biomedicines. 2021;9(5):467.

12. Adak A, Khan MR. An insight into gut microbiota and its functionalities. Cell Mol Life Sci. 2019;76(3):473-93.

13. Lee SH. Intestinal permeability regulation by tight junction: implication on inflammatory bowel diseases. Intest Res. 2015;13(1):11-8.

14. Wilson CL et al. Regulation of intestinal alpha-defensin activation by the metalloproteinase matrilysin in innate host defense. Science. 1999;286(5437):113-7.

15. Boneca IG et al. A critical role for peptidoglycan $\mathrm{N}$-deacetylation in
Listeria evasion from the host innate immune system. Proc Natl Acad Sci USA. 2007;104(3):997-1002.

16. Macpherson AJ, Uhr T. Induction of protective IgA by intestinal dendritic cells carrying commensal bacteria. Ann NY Acad Sci. 2004;1029:36-43.

17. Ivanov || et al. Induction of intestinal Th17 cells by segmented filamentous bacteria. Cell. 2009;139(3):485-98.

18. Atarashi $\mathrm{K}$ et al. Induction of colonic regulatory $T$ cells by indigenous Clostridium species. Science. 2011;331(6015):337-41.

19. Guo TL et al. Gut microbiome in neuroendocrine and neuroimmune interactions: the case of genistein. Toxicol Appl Pharmacol. 2020:402:115130.

20. Ferreira RDS et al. Relationship between intestinal microbiota, diet and biological systems: an integrated view. Crit Rev Food Sci Nutr. 2020;29:1-21.

21. Kitching $A R$, Holdsworth SR. The emergence of TH17 cells as effectors of renal injury. J Am Soc Nephrol. 2011:22(2):235-8.

22. Andrade-Oliveira $\mathrm{V}$ et $\mathrm{al}$. Inflammation in renal diseases: new and old players. Front Pharmacol. 2019;10:1192.

23. Noel S et al. Intestinal microbiotakidney cross talk in acute kidney injury and chronic kidney disease. Nephron Clin Pract. 2014;127(1-4):13943.

24. Cigarran Guldris S et al. Gut microbiota in chronic kidney disease. Nefrologia. 2017;37(1):9-19.

25. Swarte JC et al. Characteristics and dysbiosis of the gut microbiome in renal transplant recipients. J Clin Med. 2020;9(2):386.

26. Lee JR et al. Gut microbial community structure and complications after kidney transplantation: a pilot study. Transplantation. 2014;98(7):697-705.

27. Westblade LF et al. Gastrointestinal pathogen colonization and the microbiome in asymptomatic kidney transplant recipients. Transpl Infect Dis. 2019;21(6):e13167.

28. Wang W et al. Gut microbiota and allogeneic transplantation. J Transl Med. 2015;13:275

29. Fricke WF et al. Human microbiota characterization in the course of renal transplantation. Am J Transplant. 2014;14(2):416-27.
30. Anders $\mathrm{HJ}$ et al. The intestinal microbiota, a leaky gut, and abnorma immunity in kidney disease. Kidney Int. 2013;83(6):1010-6.

31. Bromberg JS et al. Microbiota - implications for immunity and transplantation. Nat Rev Nephrol. 2015;11(6):342-53.

32. Evenepoel $\mathrm{P}$ et al. The gut-kidney axis. Pediatr Nephrol. 2017; 32(11):2005-14.

33. Ardalan M, Vahed SZ. Gut microbiota and renal transplant outcome. Biomed Pharmacother. 2017;90:229 36.

34. Chan S et al. Transplant associated infections - the role of the gastrointestinal microbiota and potential therapeutic options. Nephrology (Carlton). 2020;25(1):513.

35. Carron $\mathrm{C}$ et al. End-stage renal disease-associated gut bacterial translocation: evolution and impact on chronic inflammation and acute rejection after renal transplantation. Front Immunol. 2019;10:1630.

36. Wang J et al. Gut microbiota alterations associated with antibodymediated rejection after kidney transplantation. Appl Microbiol Biotechnol. 202;105(6):2473-84.

37. Wolfe AJ et al. Evidence of uncultivated bacteria in the adult female bladder. J Clin Microbiol. 2012;50(4):1376-83.

38. Whiteside SA et al. The microbiome of the urinary tract - a role beyond infection. Nat Rev Urol. 2015;12(2):8190.

39. Pearce $M M$ et al. The female urinary microbiome: a comparison of women with and without urgency urinary incontinence. MBio. 2014;5(4):e01283 14

40. Lee JR et al. Butyrate-producing gut bacteria and viral infections in kidney transplant recipients: a pilot study. Transpl Infect Dis. 2019;21(6):e13180.

41. Biehl LM et al. Fecal microbiota transplantation in a kidney transplant recipient with recurrent urinary tract infection. Infection. 2018;46(6):871-4.

42. Modena BD et al. Changes in urinary microbiome populations correlate in kidney transplants with interstitial fibrosis and tubular atrophy documented in early surveillance biopsies. Am J Transplant. 2017:17(3):712-23.

43. Xiao $\mathrm{J}$ et al. Organ transplantation 
and gut microbiota: current reviews and future challenges. Am J Transl Res. 2018;10(11):3330-44.

44. Lee JR et al. Gut microbiota dysbiosis and diarrhea in kidney transplant recipients. Am J Transplant. 2019:19(2):488-500.

45. Rajilić-Stojanović M, de Vos WM. The first 1000 cultured species of the human gastrointestinal microbiota. FEMS Microbiol Rev. 2014; 38(5):9961047.

46. Zhang LT et al. Gut microbiota profiles and fecal beta-glucuronidase activity in kidney transplant recipients with and without post-transplant diarrhea. Clin Transplant. 2021:e14260.

47. Kelly CR et al. Effect of fecal microbiota transplantation on recurrence in multiply recurrent clostridium difficile infection: a randomized trial. Ann Intern Med. 2016;165(9):609-16.

48. Wu H et al. Gut microbial metabolites induce donor-specific tolerance of kidney allografts through induction of $\mathrm{T}$ regulatory cells by short-chain fatty acids. J Am Soc Nephrol. 2020;31(7):1445-61.

49. Andrade-Oliveira $\vee$ et al. Gut bacteria products prevent $\mathrm{AKI}$ induced by ischemia-reperfusion. J Am Soc Nephrol. 2015;26(8):1877-88.

50. Colas $L$ et al. Unique and specific proteobacteria diversity in urinary microbiota of tolerant kidney transplanted recipients. Am J Transplant. 2020;20(1):145-58.
51. Poesen $\mathrm{R}$ et al. The influence of renal transplantation on retained microbialhuman co-metabolites. Nephrol Dial Transplant. 2016;31(10):1721-9.

52. Zheng $Y$ et al. Identification of antibiotic administration as a potentially novel factor associated with tacrolimus trough variability in kidney transplant recipients: a preliminary study. Transplant Direct. 2019;5(9):e485.

53. Lee JR et al. Gut microbiota and tacrolimus dosing in kidney transplantation. PLoS One. 2015;10(3):e0122399.

54. Guo Y et al. Commensal gut bacteria convert the immunosuppressant tacrolimus to less potent metabolites Drug Metab Dispos. 2019;47(3):194 202

55. Gibson CM et al. The alteration of the gut microbiome by immunosuppressive agents used in solid organ transplantation. Transpl Infect Dis. 2021:23(1):e13397.

56. Nallu A et al. Gut microbiome in chronic kidney disease: challenges and opportunities. Transl Res. 2017;179:24-37

57. Thomas CM, Versalovic J. Probioticshost communication: modulation of signaling pathways in the intestine. Gut Microbes. 2010;1(3):148-63.

58. Maudet $\mathrm{C}$ et al. MicroRNAs in the interaction between host and bacterial pathogens. FEBS Lett. 2014:588(22):4140-7.

59. Rayes $\mathrm{N}$ et al. Supply of pre- and probiotics reduces bacterial infection rates after liver transplantation - a randomized, double-blind trial. Am J Transplant. 2005;5(1):125-30.

60. Eguchi $\mathrm{S}$ et al. Perioperative synbiotic treatment to prevent infectious complications in patients after elective living donor liver transplantation: a prospective randomized study. Am J Surg. 2011;201(4):498-502.

61. Rayes $\mathrm{N}$ et al. Early enteral supply of lactobacillus and fiber versus selective bowel decontamination: a controlled trial in liver transplant recipients. Transplantation. 2002;74(1):123-7

62. Slavin J. Fiber and prebiotics: mechanisms and health benefits. Nutrients. 2013; 5(4):1417-35.

63. Markowiak P, Śliżewska K. Effects of probiotics, prebiotics, and synbiotics on human health. Nutrients. 2017;9(9):1021.

64. van Nood E et al. Duodenal infusion of feces for recurrent Clostridium difficile. N Engl J Med. 2013;368(22):2145

65. Henig I et al. The clinical role of the gut microbiome and fecal microbiota transplantation in allogeneic stem cell transplantation. Haematologica. 2021;106:933-46.

66. Wang $Z$ et al. Non-lethal inhibition of gut microbial trimethylamine production for the treatment of atherosclerosis. Cell. 2015 163(7):1585-95. 